\title{
Analysis of the Immunogenicity of mRNA-1273 SARS-CoV-2 Vaccine in Breast Cancer Patients Treated With Cyclin-Dependent Kinases 4 and 6 Inhibitors (CDKi): Limited T-Cell Immunity Despite Satisfactory Humoral Response.
}

\section{Cristina Saavedra}

Ramon y Cajal Universitary Hospital https://orcid.org/0000-0002-1063-2306

Alejandro Vallejo

Ramon y Cajal Universitary Hospital

\section{Federico Longo}

Ramon y Cajal Universitary Hospital https://orcid.org/0000-0003-0902-2188

Juan José Serrano

Ramon y Cajal Universitary Hospital https://orcid.org/0000-0003-4126-7150

María Fernández

Ramon y Cajal Universitary Hospital

Maria Gion

Ramon y Cajal University Hospital

Elena López-Miranda

Ramon y Cajal Universitary Hospital

Noelia Martinez-Jañez

Ramon y Cajal Universitary Hospital

\section{Eva Guerra}

Ramon y Cajal Universitary Hospital

Jesús Chamorro

Ramon y Cajal Universitary Hospital

\section{Diana Rosero}

Ramon y Cajal Universitary Hospital

Héctor Velasco

Ramon y Cajal Universitary Hospital

Adrian Martin

Ramon y Cajal Universitary Hospital

Alfredo Carrato

Ramon y Cajal Universitary Hospital 
Jose Luis Casado

Ramon y Cajal Universitary Hospital https://orcid.org/0000-0002-7639-8591

Alfonso Cortes ( $\square$ acsalgado86@gmail.com )

Ramon y Cajal Universitary Hospital

\section{Brief Communication}

Keywords: Analysis, SARS-CoV-2 vaccine, breast cancer patients, cyclin-dependent kinases, inhibitors, Tcell immunity, humoral response, immunogenicity

Posted Date: September 28th, 2021

DOl: https://doi.org/10.21203/rs.3.rs-798329/v1

License: (c) (i) This work is licensed under a Creative Commons Attribution 4.0 International License.

Read Full License 


\section{Abstract}

SARS-CoV-2 vaccination in cancer patients has become crucial because of their higher risk of complications and death from COVID19. We performed a prospective study to assess the immunogenicity of SARS-CoV-2 vaccine in cancer patients treated with CDKi and compared their immune response with patients treated with chemotherapy. While a robust humoral response was observed in the CDKi patients compared with chemotherapy patients, limited T-cell immunity was achieved in both groups.

\section{Introduction}

COVID19 pandemic spread globally leading to 187 million cases and 4 million deaths throughout the world [1]. Cancer patients are a high risk population due to the immunosuppression linked to disease and therapies. The emergence of SARS-CoV-2 vaccines supposed an important step in pandemic control. Since cancer patients were not included in vaccines pivotal trials, concerning about safety and efficacy arose too. Currently, we can find some small prospective in cancer patients, which conclude that solid tumour patients need at least two doses to achieve a satisfactory humoral response, whereas haematological patients show worse results [2-7].

To our knowledge, there are not specific data about COVID19 immunization in patients on therapy with CDKi. CDKi in combination with hormonal therapies constitute the standard treatment in luminal metastatic breast cancer [8]. Secondary neutropenia occurs in up to $80 \%$ of patients, being grade $3-4$ in $50 \%$ and $10 \%$ of cases, respectively [9]. This toxicity profile could influence vaccination immune response.

We present our analysis of the efficacy of mRNA-1273 vaccine in patients treated with CDKi, compared with the results obtained in patients treated with chemotherapy.

\section{Methods}

We conducted a prospective unicentric study in patients with solid malignancies. The study was performed in accordance with Good Clinical Practice guidelines and the World Medical Association Declaration of Helsinki. It was approved by the institutional review board of Ramón y Cajal University Hospital (IRB-21) and all patients provided written informed consent before any procedure.

At baseline, and at least 17 days after the first and second dose of vaccine, approximately $30 \mathrm{~mL}$ of venous blood were obtained in EDTA tubes. Specific antibodies were assessed in the three successive samples against the S domain of the spike protein (SARS-CoV-2 IgG II Quant Alinity; Abbott, Maidenhead, UK; positivity threshold 50 arbitrary units $(\mathrm{AU}) / \mathrm{ml}$ ). Cellular immune response was assessed at inclusion and after complete vaccination. Briefly, SARS-CoV-2-specific CD4+ and CD8+ T-cells were measured using in vitro stimulation with SARS-CoV-2 peptide pools of viral proteins encompassing the spike (S), membrane (M), and nucleocapsid (N), followed by quantitation of CD4+ and CD8+ T-cell specific 
interferon (IFN)- $y$ in live cell flow cytometry, using peripheral blood mononuclear cell (PBMC) samples from all subjects. It was considered reactive if the proportion of positive cells in stimulated wells was at least 2-fold higher in comparison with the negative control wells (unstimulated).

GraphPadPrism version 8.0 was used to generate the figures and STATA-15 software to data analysis. Fisher exact test was used to compare proportions and Mann-Whitney test to compare quantitative variables. Results with a $p$ value less than 0.05 were considered statistically significant.

\section{Results}

We carried out an observational prospective study in patients with solid malignancies on active therapy, who received mRNA-1273 SARS-CoV-2 vaccine. Patients with history of previous COVID19 were excluded.

A baseline determination of humoral and T-cell response was performed and repeated at least 17 days after the first vaccine dose (1D) (humoral) and the second vaccine dose (2D) (humoral and cellular). From the 35 patients included in each cohort, 9 patients in the CDKi cohort and 3 patients in the chemotherapy cohort had a positive basal serology; and they were excluded for further analysis. Due to performance status worsening, 1D sample was not obtained in one patient from each group, and 2D sample was not obtained in 2 additional patients from the chemotherapy group (Fig. 1A). Therefore, the following comparisons were made with $26 \mathrm{CDKi}$ and 32 chemotherapy patients.

Patient characteristics are shown in Table I. In the CDKi group, the three approved drugs were well represented: abemaciclib (9 patients), palbociclib (15 patients) and ribociclib (2 patients).

The proportion of patients with positive serology was significantly higher with CDKi after 1D (100\% vs $64.5 \%, \mathrm{p} 0.001)$, without significant differences after 2D (100\% vs $89.7 \%, \mathrm{p} 0.2)$. Besides CDKi patients achieved significantly higher levels of anti-S IgG after 1D ( $p$ 0.0002) and 2D (p 0.02) (Fig. 1B). There was not a significant correlation between the humoral response and the cellular immunogenicity at baseline.

Regarding cellular immunogenicity, we found no significant differences between groups in the proportion of patients with anti-S T-cell CD4 response after 2D (69.2\% vs $51.7 \%$, p 0.3). However, positive anti-S CD8 samples were significantly higher with CDKi $(69.2 \%$ vs $37.9 \%, \mathrm{p} 0.03)$. As observed with the humoral response, the magnitude of T-cell response was higher with CDKi in comparison with chemotherapy ( $p$ 0.049 and 0.006 for CD4 and CD8 T-cell response, respectively) (Fig. 1C and 1D).

Of note, nearly one third of patients with CDKi had T-cell response at baseline, suggesting the presence of cross-reactivity in absence of prior disease or positive serology. We evaluated the response excluding those patients with basal T-cell response from the analysis ( 9 from CDKi and 5 from chemotherapy). Humoral immunogenicity remained significantly higher with CDKi both after 1D ( $p$ 0.0004) and 2D ( $p$ 0.04). Similar results were obtained regarding T-cell response: no significant differences in CD4 response (64.7 vs 44\%; p 0.1), and better CD8 response with CDKi (58.8 vs 32\%; $p 0.04$ ). 


\section{Discussion}

Immunosuppression secondary to cancer and oncologic therapies increases the risk of severe COVID19 and, at the same time, it decreases the effect of the principal weapons we have: vaccines. However, cancer patients are a heterogeneous group and specific data about each neoplasm and therapy is not available. We conducted a prospective study to analyse humoral and cellular response to vaccination in breast cancer patients receiving treatment with CDKi in comparison with patients on chemotherapy.

We have observed that patients with CDKi showed high rates of humoral response after even only one dose. Nevertheless, only $60 \%$ of patients on chemotherapy showed seroconversion after the first dose, highlighting the need of two doses in this population. Also, the anti-S titres were significantly higher with $\mathrm{CDKi}$, a relevant issue since there is a correlation of the serology with neutralizing antibodies [10]. On the other hand, higher rates of specific T-cell CD8 response were observed in patients on CDKi treatment compared with chemotherapy, but limited if we compared them with historical healthy controls [11].

It should be noted that there was a lack of correlation between humoral and cellular responses. Despite the higher rates of seroconversion in patients with CDKi, only two thirds of them had T-cell response more than 3 weeks after a correct vaccination scheme. In addition, T-cell response data with chemotherapy got worse. Since cellular immunity could be the main line of defence in presence of viral variants these data are concerning [12-14].

Mechanism underlying neutropenia with CDKi is different from chemotherapy. In fact, despite the high rates of grade 3-4 neutropenia with CDKi, the rate of infections and febrile neutropenia are extremely low [9]. Therefore, we expected to find a better immune response to vaccines with CDKi. While humoral response has been satisfactory, $T$ cell response rate is less than expected. These results are contradictory with recent data about immunomodulatory effects of CDKi. Preclinical studies showed that CDKi boost effector T-cell functions, and their combination with anti-PD-1 antibodies have synergic effect, increasing interferon production in CD8-T-cells and IL-2 in CD4-T-cells $[15,16]$. This generates the hypothesis that immunosuppression secondary to advanced neoplasms underlies the limited T-cell response, and the stimulation of cytotoxic T-cell response of CDKi would only reverse partially this effect [17].

Our study has several limitations. On one hand, the small number of patients included and the lack of data about the level of protection against SARS-CoV-2 infection and severe COVID19. On the other hand, we do not include neutralizing antibodies evaluation that could be responsible of protection even with lack of adequate T-cell response and they have been correlated with specific antibodies [10]. Also, we include patients with cross-reactive cellular immunity at baseline that could have improved the final rate of T-cell response $[18,19]$.

In conclusion, humoral response is adequate in patients treated with CDKi and quantitatively lower with chemotherapy. However, cellular response is low in both groups, especially in patients on chemotherapy. These data should be considered in the vaccination schedules, and new vaccination strategies should be developed for cancer patients. 


\section{Declarations}

\section{Data availability}

The data generated and analysed during this study are described in the data record. The immune response and clinical data are available in the Excel spreadsheet "BD_ONCO_300621.xIsx", which is openly available and shared as part of the figshare data record. The "ONCOVAC study" dataset is not publicly available to protect patient privacy. Requests for access to this dataset can be made to the corresponding author.

\section{Conflict of interest:}

For the purpose of this article:

Cristina Saavedra: Travel and educational support: Novartis, Lilly and Pfizer.

Alfonso Cortés: Speaker's bureau: Novartis. Research funding: Pfizer.

María Fernández: Advisory/consultancy honorarium: Lilly. Speaker bureau/expert testimony honorarium: Pfizer, Lilly, Novartis. Travel/accommodation/expenses: Pfizer. Research funding: Pfizer. Other economic interest in a private business related with health care: soluciones de bienestar para pacientes oncologicos RIFECO SL.

María Gion: Travel and educational support: Novartis and Pfizer.

Elena López: Advisory/consultancy honorarium: Pfizer, Novartis. Speaker bureau/expert testimony honorarium: Novartis. Travel/accommodation/expenses: Novartis.

Noelia Martínez: Advisory board: Novartis, Pfizer and Lilly.

Alfredo Carrato: Honoraria as collaboration in advisory boards: Roche, Merck, MSD, Servier, Bayer, Baxter and BMS.

Other authors declare not conflict of interest for the purpose of this article.

*Author contributions: Cristina Saavedra, Alejandro Vallejo, Federico Longo and Juan José Serrano equally contributed to this work.

\section{References}

1. WHO COVID-19 Dashboard. Geneva: World Health Organization, 2020. Available online: https://covid19.who.int/.

2. Addeo A, Shah PK, Bordry N, Hudson RD, Albracht B, Di Marco M, et al. Immunogenicity of SARS-CoV-2 messenger RNA vaccines in patients with cancer. Cancer cell. 2021; 
3. Shroff RT, Chalasani P, Wei R, Pennington D, Quirk G, Schoenle M V, et al. Immune Responses to COVID19 mRNA Vaccines in Patients with Solid Tumors on Active, Immunosuppressive Cancer Therapy. medRxiv: the preprint server for health sciences. 2021.

4. Barrière J, Chamorey E, Adjtoutah Z, Castelnau O, Mahamat A, Marco S, et al. Impaired immunogenicity of BNT162b2 anti-SARS-CoV-2 vaccine in patients treated for solid tumors. Annals of oncology: official journal of the European Society for Medical Oncology. 2021. p. 1053-5.

5. Palich R, Veyri M, Marot S, Vozy A, Gligorov J, Maingon P, et al. Weak immunogenicity after a single dose of SARS-CoV-2 mRNA vaccine in treated cancer patients. Annals of oncology: official journal of the European Society for Medical Oncology. 2021. p. 1051-3.

6. Monin L, Laing AG, Muñoz-Ruiz M, McKenzie DR, Del Molino Del Barrio I, Alaguthurai T, et al. Safety and immunogenicity of one versus two doses of the COVID-19 vaccine BNT162b2 for patients with cancer: interim analysis of a prospective observational study. The Lancet Oncology. 2021;22:765-78.

7. Mansi L, Spehner L, Daguindau E, Bouiller K, Almotlak H, Stein U, et al. Study of the SARS-CoV-2specific immune T-cell responses in COVID-19-positive cancer patients. European journal of cancer (Oxford, England: 1990). 2021;150:1-9.

8. Cardoso F, Paluch-Shimon S, Senkus E, Curigliano G, Aapro MS, André F, et al. 5th ESO-ESMO international consensus guidelines for advanced breast cancer ( $A B C 5$ ). Annals of oncology : official journal of the European Society for Medical Oncology. 2020. p. 1623-49.

9. Spring LM, Zangardi ML, Moy B, Bardia A. Clinical Management of Potential Toxicities and Drug Interactions Related to Cyclin-Dependent Kinase 4/6 Inhibitors in Breast Cancer: Practical Considerations and Recommendations. The oncologist. 2017;22:1039-48.

10. Wu F, Wang A, Liu M, Wang Q, Chen J, Xia S, et al. Neutralizing antibody responses to SARS-CoV-2 in a COVID-19 recovered patient cohort and their implications. medRxiv. 2020;2020.03.30.20047365.

11. Painter MM, Mathew D, Goel RR, Apostolidis SA, Pattekar A, Kuthuru O, et al. Rapid induction of antigen-specific CD4\&lt;sup\&gt;+\&lt;/sup\&gt; T cells guides coordinated humoral and cellular immune responses to SARS-CoV-2 mRNA vaccination. bioRxiv. 2021;2021.04.21.440862.

12. Goletti D, Petrone L, Manissero D, Bertoletti A, Rao S, Ndunda N, et al. The potential clinical utility of measuring SARS-CoV-2-specific T-cell responses. Clinical microbiology and infection: the official publication of the European Society of Clinical Microbiology and Infectious Diseases. 2021;

13. Geers D, Shamier MC, Bogers S, den Hartog G, Gommers L, Nieuwkoop NN, et al. SARS-CoV-2 variants of concern partially escape humoral but not T-cell responses in COVID-19 convalescent donors and vaccinees. Science immunology. United States; 2021;6. 
14. Woldemeskel BA, Garliss CC, Blankson JN. SARS-CoV-2 mRNA vaccines induce broad CD4+ T cell responses that recognize SARS-CoV-2 variants and HCoV-NL63. The Journal of clinical investigation. $2021 ; 131$.

15. Petroni G, Formenti SC, Chen-Kiang S, Galluzzi L. Immunomodulation by anticancer cell cycle inhibitors. Nature reviews Immunology. 2020;20:669-79.

16. Deng J, Wang ES, Jenkins RW, Li S, Dries R, Yates K, et al. CDK4/6 Inhibition Augments Antitumor Immunity by Enhancing T-cell Activation. Cancer discovery. 2018;8:216-33.

17. Eberl G. Immunity by equilibrium. Nature reviews Immunology. England; 2016;16:524-32.

18. Loyal L, Braun J, Henze L, Kruse B, Dingeldey M, Reimer U, et al. Cross-reactive CD4\&lt;sup\&gt;+\&lt;/sup\&gt; $T$ cells enhance SARS-CoV-2 immune responses upon infection and vaccination. medRxiv. 2021;2021.04.01.21252379.

19. Le Bert N, Tan AT, Kunasegaran K, Tham CYL, Hafezi M, Chia A, et al. SARS-CoV-2-specific T cell immunity in cases of COVID-19 and SARS, and uninfected controls. Nature. England; 2020;584:457-62.

\section{Tables}


Table I - Patient characteristics.

\begin{tabular}{|c|c|c|c|}
\hline & CDK4/6 inhibitors $(n=26)$ & Chemotherapy (n=32) & p \\
\hline Age $^{1}$ & $52.6(46.1-60.6)$ & $61.2(55.8-69.0)$ & 0.002 \\
\hline Female & $100 \%$ & $65.6 \%$ & 0.001 \\
\hline Caucasian & $100 \%$ & $96.9 \%$ & 1 \\
\hline \multicolumn{4}{|l|}{ Primary tumour } \\
\hline - Breast & $100 \%$ & $25 \%$ & $<0.001$ \\
\hline - Non-colorectal GI cancer & & $37.5 \%$ & \\
\hline - Colorectal cancer & & $25 \%$ & \\
\hline - Ovarian cancer & & $9.4 \%$ & \\
\hline - cup & & $3.1 \%$ & \\
\hline Stage IV & $96.2 \%$ & $78.1 \%$ & 0.06 \\
\hline \multicolumn{4}{|l|}{ White cells at $B^{1}$} \\
\hline - Leucocytes & $3415(3070-4800)$ & $5015(3705-6260)$ & 0.01 \\
\hline - Neutrophils & $1855(1180-1960)$ & $2905(1695-4215)$ & 0.002 \\
\hline - Lymphocytes & $1420(1140-2500)$ & $1360(1045-1835)$ & 0.5 \\
\hline \multicolumn{4}{|l|}{ Humoral immunogenicity (anti-S IgG) ${ }^{1}$} \\
\hline \multirow[t]{2}{*}{ - 1D } & $2567.4(777-5644.3)$ & $263.9(15.1-983.2)$ & 0.0002 \\
\hline & $100 \%$ & $64.5 \%$ & 0.001 \\
\hline \multirow[t]{2}{*}{ - $2 \mathrm{D}$} & $23989.9(7185.7-30606.1)$ & $4293.6(2234.5-19013)$ & 0.02 \\
\hline & $100 \%$ & $89.7 \%$ & 0.2 \\
\hline \multicolumn{4}{|l|}{ T-cell cross-reactivity at BL } \\
\hline - $\quad$ CD4 & $23.1 \%$ & $9.4 \%$ & 0.3 \\
\hline - $\quad$ CD8 & $34.6 \%$ & $12.5 \%$ & 0.06 \\
\hline \multicolumn{4}{|l|}{ T-cell response after $2 \mathrm{D}$} \\
\hline \multirow[t]{2}{*}{ - $\quad$ Anti-S CD4 } & $0.07(0-0.21)$ & $0.01(0-0.08)$ & 0.049 \\
\hline & $69.2 \%$ & $51.7 \%$ & 0.3 \\
\hline \multirow[t]{2}{*}{ - $\quad$ Anti-S CD8 } & $0.09(0-0.23)$ & $0(0-0.04)$ & 0.007 \\
\hline & $69.2 \%$ & $37.9 \%$ & 0.03 \\
\hline
\end{tabular}

${ }^{\dagger}$ Data are expressed as median (interquartile range) and proportion of positive cases when indicated.

$B L=$ baseline. $1 D=$ after first vaccine dose. $2 D=$ after second vaccine dose.

\section{Figures}


A

Study flow diagram
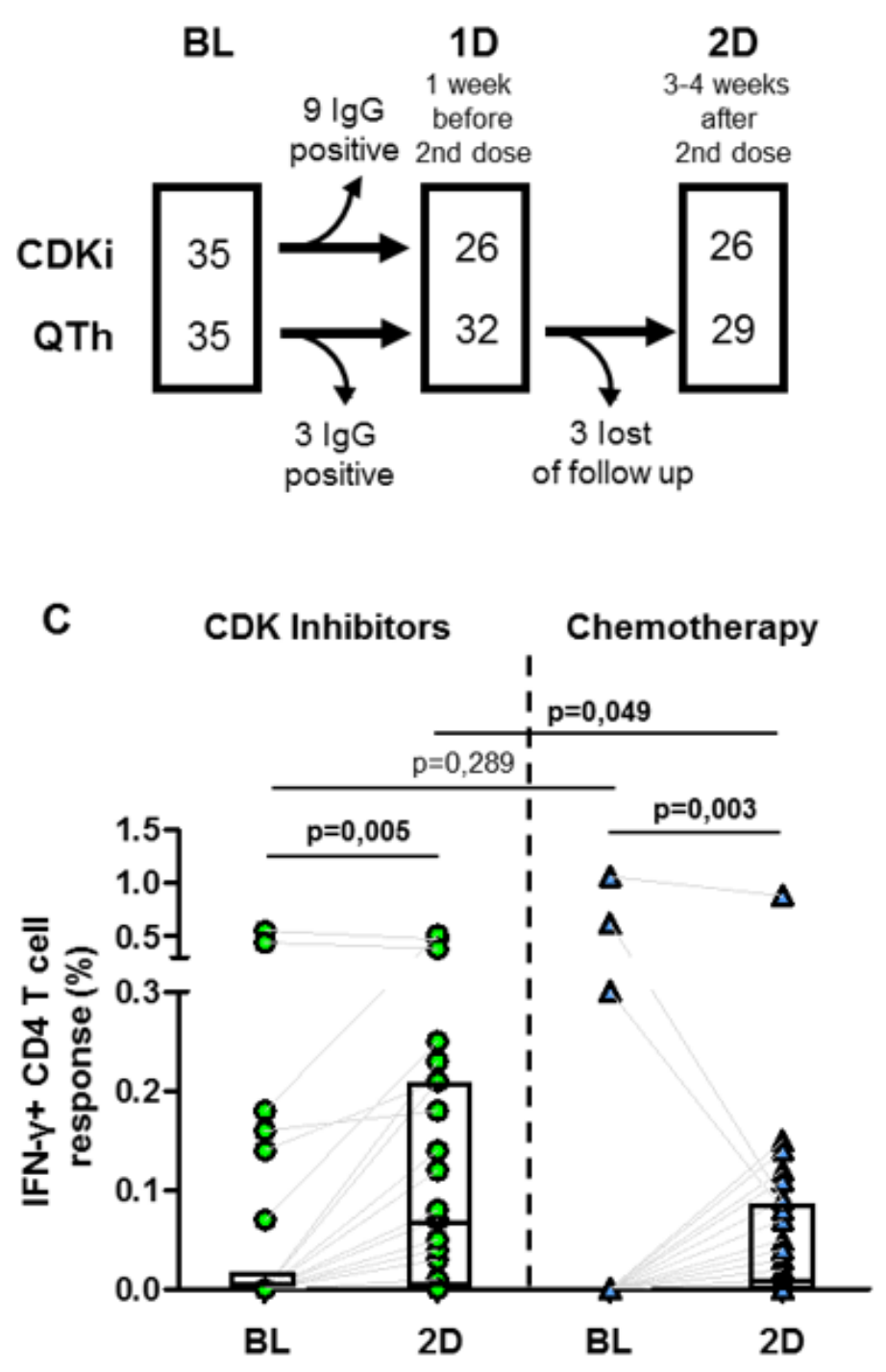
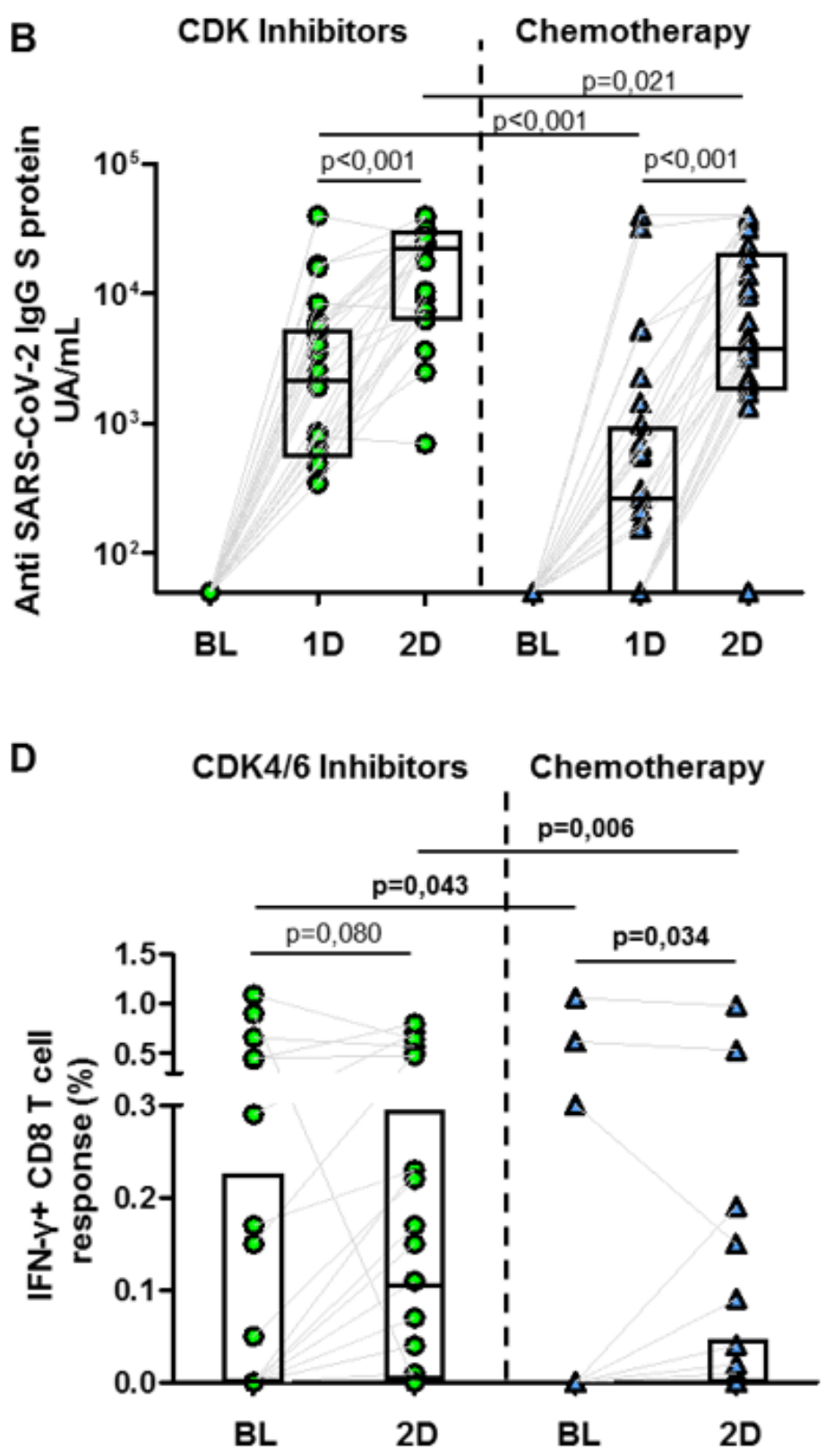

\section{Figure 1}

1D sample was not obtained in one patient from each group, and 2D sample was not obtained in 2 additional patients from the chemotherapy group (Figure 1A). Besides CDKi patients achieved significantly higher levels of anti-S IgG after 1D ( $p$ 0.0002) and 2D ( $p$ 0.02) (Figure 1B). As observed with the humoral response, the magnitude of T-cell response was higher with CDKi in comparison with chemotherapy (p 0.049 and 0.006 for CD 4 and CD8 T-cell response, respectively) (Figure 1C and 1D). 